\title{
USO POTENCIAL DA CASCA DE ARROZ CARBONIZADA NA COMPOSIÇÃO DE SUBSTRATOS PARA PRODUÇÃO DE MUDAS DE Anadenanthera peregrina (L) Speg.
}

Potential use of carbonized rice bark in composition of substrates for the production of changes of Anadenanthera peregrina ( $L)$ Speg.

Uso potencial de la casca de arroz carbonizada en la composición de substratos para la producción de cambios de Anadenanthera peregrina (L) Speg.

\section{Euclides Figueredo Fonseca*1, Gabriel Oliveira Silva ${ }^{1}$, David Lucas Camargo Vieira Terra $^{1}$, Priscila Bezerra de Souza ${ }^{1}$ \\ ${ }^{1}$ Curso de Engenharia Florestal, Universidade Federal do Tocantins, Gurupi, Tocantins, Brasil.}

*Correspondência: Universidade Federal do Tocantins, UFT - Rua Badejós, Lt. 07 Ch. 69/72 Zona Rural 77402970 - Gurupi, TO - Brasil - Caixa-postal: 66.e-mail euclidesgpi_14@hotmail.com

\section{RESUMO}

Objetivou-se avaliar a influência da casca de arroz carbonizada como substrato na produção de mudas de angicovermelho Anadenanthera peregrina $(\mathrm{L})$ Speg. O experimento foi desenvolvido no Viveiro Florestal da Universidade Federal do Tocantins, campus de Gurupi, sendo conduzido em delineamento inteiramente casualizado, sendo constituído por 5 tratamentos (T1: 0\% de Casca de Arroz Carbonizada (CAC) + 100\% de Substrato comercial; T2: 25\% de CAC $+75 \%$ de Substrato comercial; T3: $50 \%$ de CAC $+50 \%$ de Substrato comercial; T4: 75\% de CAC + 25\% de Substrato comercial; T5: 100\% de CAC + 0\% de Substrato comercial), com 3repetições, onde em cada repetição foram avaliadas as médias de 10 plântulas. De modo geral, observou-se um efeito da adição da casca de arroz carbonizada ao substrato comercial no desenvolvimento da espécie Anadenanthera peregrina, ou seja, mudas de Anadenanthera peregrina produzidas com altas proporções de casca de arroz carbonizada ao substrato comercial tiveram uma boa resposta (Efeito positivo) no seu desenvolvimento. Portanto recomenda-se utilizar 100\% de casca de arroz carbonizada $+0 \%$ de Substrato comercial, pois esse tratamento permitiu melhor desempenho germinativo, mostrando-se mais adequados para a produção eficaz de mudas da espécie.

Palavras-chave: Angico-vermelho, sementes florestais, índice de qualidade de Dickson.

\section{ABSTRACT}

The objective of this study was to evaluate the influence of the charred rice bark as substrate on the production of angus red seedlings Anadenanthera peregrina (L) Speg. The experiment was carried out in the Forest Nursery of the Federal University of Tocantins, Campus of Gurupi, and was conducted in a completely randomized design, consisting of 5 treatments (T1: 0\% of Carbonized Rice Peel (CAC) + 100\% commercial Substrate; : 25\% of CAC + 75\% of Commercial Substrate, T3: 50\% of CAC + 50\% of Commercial Substrate, T4: 75\% of CAC + 25\% of Commercial Substrate, T5: 100\% CAC + 0\% of Commercial Substrate), With 3 replications, where in each replicate the mean values of 10 seedlings were evaluated. In general, an effect of the addition of the charred rice husk on the commercial substrate was observed in the development of the species Anadenanthera peregrina, that is, Anadenanthera peregrina seedlings produced with high proportions of carbonized rice husk on the commercial 
substrate had a good response (Effect) in their development. Therefore it is recommended to use 100\% of carbonized rice husk $+0 \%$ of commercial substrate, as this treatment allowed a better germinative performance, being more suitable for the effective production of seedlings of the species.

Keywords: Angico-red, forest seed, Dickson quality index.

\section{RESUMEN}

The objective of this study was to evaluate the influence of the charred rice bark as substrate on the production of angus red seedlings Anadenanthera peregrina (L) Speg. The experiment was carried out in the Forest Nursery of the Federal University of Tocantins, Campus of Gurupi, and was conducted in a completely randomized design, consisting of 5 treatments (T1: 0\% of Carbonized Rice Peel (CAC) + 100\% commercial Substrate; : 25\% of CAC + 75\% of Commercial Substrate, T3: 50\% of CAC + 50\% of Commercial Substrate, T4: 75\% of CAC + 25\% of Commercial Substrate, T5: 100\% CAC + 0\% of Commercial Substrate), With 3 replications, where in each replicate the mean values of 10 seedlings were evaluated. In general, an effect of the addition of the charred rice husk on the commercial substrate was observed in the development of the species Anadenanthera peregrina, that is, Anadenanthera peregrina seedlings produced with high proportions of carbonized rice husk on the commercial substrate had a good response (Effect) in their development. Therefore it is recommended to use $100 \%$ of carbonized rice husk $+0 \%$ of commercial substrate, as this treatment allowed a better germinative performance, being more suitable for the effective production of seedlings of the species.

Keywords: Angico-red, forest seed, Dickson quality index.

\section{INTRODUÇÃO}

O Cerrado é o segundo maior bioma da América do Sul, abrangendo uma área de 2.036.448 $\mathrm{km}^{2}$, cerca de $22 \%$ do território nacional, sendo o Bioma predominante do Planalto Central do Brasil (MMA, 2014). Possui uma enorme variedade de espécies arbóreas que poderiam ser aproveitadas racionalmente em diversos setores da economia, além da grande quantidade de espécies que estão sendo destruídas sem que antes fossem estudadas (Feitosa et al., 2011). Cuja degradação é atribuída à expansão agrícola do País, que, segundo Souza et al. (2002), vem ocorrendo de forma irresponsável, sem preocupação com o desperdício do potencial de várias espécies nativas do cerrado.

\section{Anadenanthera peregrina (L.) Speg.}

conhecida popularmente por angico-vermelho pertence à família Leguminosae-Fabaceae sub-família Mimosoideae, com ocorrência em florestas semidecíduas e em sua transição com o Bioma Cerrado está presente desde o Estado do Tocantins até o Estado do Rio de Janeiro além de ocorrer em florestas primarias e secundarias, sobre solos pedregosos, arenosos ou argilosos, porém bem drenados. A madeira dessa espécie é indicada para construção civil devido sua elevada densidade $(1,08$ $\left.\mathrm{g} / \mathrm{cm}^{3}\right)$, cuja casca é rica em tanino sendo muito utilizada em curtumes, ainda, possui potencial apícola e medicinal (Lorenzi, 2009).

A referida espécie é recomendada para a recuperação de áreas degradadas, pois contribui como poleiros para aves e consequentemente dispersão e chuva de sementes (Araújo et al., 2006; Berloffa et al., 2015; Dias et al., 2015). Entretanto a falta de conhecimento sobre ecologia, biologia, manejo e técnicas de propagação tem sido um fator limitante para a utilização de espécies nativas na recomposição ambiental (Ranieri et al., 2003).

Umas das definições de substrato para produção de mudas é o meio adequado para sua sustentação e retenção de nutrientes, oxigênio e água suficientes para seu desenvolvimento, ausência de elementos tóxicos, CTC adequada e pH entre 6 e 6,5 (Rosa JR. et al., 1998; Smiderle et al., 2001; Navroski 
et al., 2016; Freitas et al., 2017; Vendruscolo et al., 2017).

Vários materiais podem ser utilizados como substratos e tem por finalidade garantir o desenvolvimento da planta, levando em consideração a disponibilidade, o custo e as características físicoquímicas do mesmo. Os materiais utilizados como substratos podem ser divididos em materiais orgânicos (moinha de carvão, bagaço de cana, serragem, trufas, casca de Pinus) e materiais inorgânicos (areia, perlita, rochas vulcânicas entre outros). Um material orgânico que vem sendo utilizado na composição de substratos para produção de mudas é a casca de arroz carbonizada.

Klein et al. (2002) e Melo et al. (2014) observaram que quando misturada a substratos comerciais em diferentes proporções, a casca de arroz carbonizada propiciava melhor porosidade, podendo ser utilizada para melhorar as características físicohídricas dos substratos.

Desta forma, a casca de arroz quando carbonizada apresenta fácil manuseio, alta capacidade de drenagem, peso reduzido, teores de $\mathrm{K}$ e Ca que são macronutrientes essenciais para o desenvolvimento das plântulas, além da forma floculada, $\mathrm{pH}$ levemente alcalino e ser livre de patógenos e nematoides (Tabajara et al., 1986; Favalessa, 2011). Segundo Minami (1995), a casca de arroz carbonizada possui características que associadas ao seu baixo custo de transporte devido a sua leveza, e por se tratar do aproveitamento de um material que a princípio era visto como negativo ao meio ambiente, torna-se interessante e curioso para a avaliação desse material como componente de substrato alternativo (Bezerra et al., 2017).

Portanto conhecer as condições que proporcionem germinação rápida e uniforme das sementes de espécies nativas é extremamente importante no momento da semeadura. Já que a germinação rápida e o desenvolvimento homogêneo das plântulas reduzem os cuidados advindos dos viveiristas, uma vez que as mudas terão o desenvolvimento mais acelerado, resultando em um povoamento uniforme no campo (Pacheco et al., 2006).

Diante do contexto, objetivou-se avaliar a influência da casca de arroz carbonizada como substrato alternativo na produção de mudas da espécie Anadenanthera peregrina (L) Speg. conhecida popularmente por angico-vermelho.

\section{MATERIAIS E MÉTODOS}

O experimento foi desenvolvido no período de novembro de 2015 a fevereiro de 2016, sendo o mesmo conduzido no Viveiro Florestal da Universidade Federal do Tocantins, Campus Universitário de Gurupi-TO, a $280 \mathrm{~m}$ de altitude, sob as coordenadas $11^{\circ} 43^{\prime} 45^{\prime}$ ' de latitude Sul e $49^{\circ}$ 04'07', de longitude Oeste.

O clima da região segundo Köppen é do tipo AW, definido como tropical úmido com estação chuvosa no verão e seca no inverno. A precipitação média anual varia entre $1.500 \mathrm{~mm}$ a $1.600 \mathrm{~mm}$ e temperatura média ao longo do ano entre $22^{\circ} \mathrm{C}$ e $28^{\circ} \mathrm{C}$ (Klink e Machado, 2005).

Os frutos de Anadenanthera peregrina (L) Speg. foram colhidos em 5 árvores matrizes em um fragmento de Cerrado localizado na Universidade Federal do Tocantins, Campus de Gurupi, em seguida foram transportadas até o Laboratório de Sementes Florestais, onde as sementes foram beneficiadas e selecionadas manualmente, descartando-se as que apresentavam injúrias ou deformações.

O trabalho foi dividido em 5 tratamentos (T1: $0 \%$ de Casca de Arroz Carbonizada (CAC) $+100 \%$ de Substrato comercial; T2: $25 \%$ de CAC $+75 \%$ de Substrato comercial; T3: $50 \%$ de CAC $+50 \%$ de Substrato comercial; T4: $75 \%$ de CAC $+25 \%$ de 
Substrato comercial; T5: $100 \%$ de CAC $+0 \%$ de Substrato comercial) o substrato comercial utilizado foi o Bioflora $^{\circledR}$. Os mesmos foram implantados em delineamento experimental inteiramente casualizado com 3 repetições sendo que em cada repetição foram avaliadas as médias de 10 plântulas. Após o plantio foram realizadas duas irrigações diárias em sistema de micro aspersão.

A altura da parte aérea foi determinada aos 30, 60 e 90 dias após a emergência (DAE) a partir do nível do substrato até a ponta da última folha, o diâmetro do coleto também foi aferido aos 30, 60 e 90 DAE ao nível do substrato. As determinações dos pesos de matéria seca da parte aérea (PMSPA) e do peso de matéria seca das raízes (PMSR) foram efetuadas a partir do material seco em estufa, regulada para $50^{\circ} \mathrm{C}$, por 72 horas.

$\mathrm{O}$ peso de matéria seca total (PMST) foi obtido pela soma dos pesos citados e as relações entre as características medidas foram determinadas pela simples divisão entre elas, ressaltando que esses cálculos foram realizados para obter-se o índice de qualidade de Dickson (IQD) que foi determinado em função da altura da parte aérea $(H)$, do diâmetro do coleto (DC), do peso de matéria seca da parte aérea (PMSPA) e do peso de matéria seca das raízes (PMSR) por meio da fórmula (DICKSON et al., 1960) apresentada a seguir:

$\mathrm{IQD}=\frac{\text { PMST }(\mathrm{g})}{\mathrm{H}(\mathrm{cm}) / \mathrm{DC}(\mathrm{mm})+\operatorname{PMSPA}(\mathrm{g}) / \operatorname{PMSR}(\mathrm{g})}$

O delineamento experimental utilizado foi o inteiramente ao acaso, consistindo de cinco tratamentos e três repetições. Os dados foram submetidos à análise de variância e as médias, comparadas pelo teste de Tukey, a 5\% de probabilidade.

\section{RESULTADOS E DISCUSSÃO}

De modo geral, observou-se no presente trabalho diferença significativa dos tratamentos testados sobre o desenvolvimento das mudas de Anadenanthera peregrina (L) Speg.

Observou-se que a que à adição de diferentes concentrações de casca de arroz carbonizada (CAC) no substrato comercial Bioflora ${ }^{\circledR}$, causou um efeito positivo no desenvolvimento das mudas de angicovermelho. Dados estes que corroboram com Saidelles et al., (2009) onde os mesmos avaliaram tratamentos semelhantes e variáveis como (H, DC, IQD) no desenvolvimento das mudas das espécies Enterolobium contortisiliquum e Apuleia leiocarpa mostrando que havia um aumento no crescimento das plântulas à medida que se aumentava a adição de CAC no solo, dessa forma, o melhor tratamento encontrado foi a mistura de $50 \%$ de CAC.

As médias de altura de plântulas da espécie Anadenanthera peregrina diferiram significativamente entre os tratamentos testados (Figura 1). Os tratamentos que proporcionaram maior crescimento de mudas, em altura, foram osT5, T2 e $\mathrm{T} 4$, os quais apresentavam em sua composição adição de 100, 25 e $75 \%$ de CAC respectivamente.

Os resultados de altura $(\mathrm{H})$ obtidos aos $60 \mathrm{e}$ 90 DAE mostrou-se diferenças significativas entre os tratamentos $(\mathrm{T} 1=4,73$ e 5,12); $(\mathrm{T} 2=5,32$ e 5,99); $(\mathrm{T} 3=$ $4,5$ e 5,63$) ;(\mathrm{T} 4=5,03$ e 5,81$)$ e (T5 $=6,28$ e 7,49$)$, ou seja, conforme aumentava a proporção de casca de arroz carbonizada no substrato comercial Bioflora ${ }^{\circledR}$ obtinha-se diferenças dos valores entre os tratamentos. Já os resultados de altura $(\mathrm{H})$ verificados aos 30 DAE não obtiveram estatisticamente diferenças entre os tratamentos testados (Figura 1). 
Teste de Tuckey Altura 60

dias

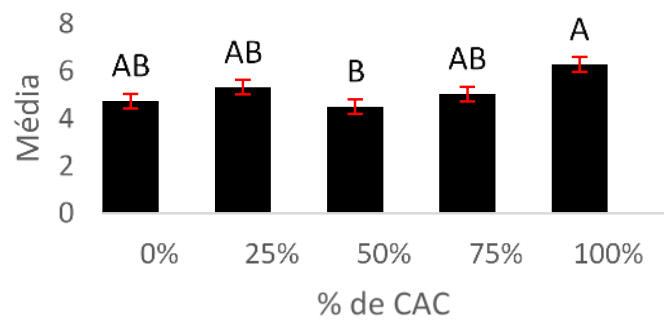

Teste de Tuckey Altura 90

dias

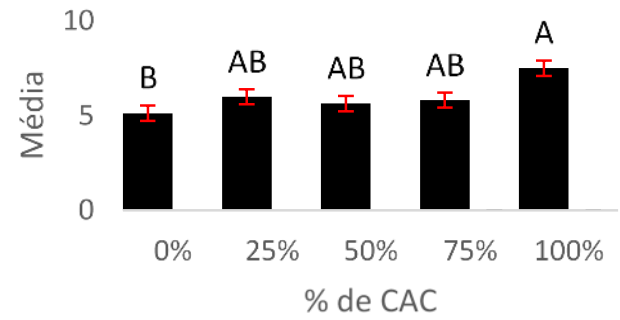

Figura 1. Teste de comparação de médias das alturas aos 60 e 90 DAE, médias seguidas pela mesma letra não diferem entre si, pelo teste de Tukey, a 5\%.

As mudas de angico-vermelho produzidas no tratamento T5 apresentaram maior diâmetro de caule $(1,61 \mathrm{~mm} ; 1,81 \mathrm{~mm}$ e $1,77 \mathrm{~mm})$ Figura 2, e as mudas dos demais tratamentos apresentaram resultados inferiores, porém iguais entre si estatisticamente. $\mathrm{O}$ diâmetro do coleto é facilmente mensurável, não sendo um método destrutivo, considerado por muitos pesquisadores um dos mais importantes parâmetros para estimar a sobrevivência logo após o plantio de mudas de diferentes espécies florestais (Gomes e Paiva, 2011; Duarte et al., 2015).

Daniel et al. (1997) e Delarmelina et al. (2015) afirmaram que o diâmetro do coleto, em geral, é o mais observado para indicar a capacidade de sobrevivência das mudas no campo, e pode auxiliar na definição das doses de fertilizantes a serem aplicadas na produção de mudas. Nesse sentido, o presente trabalho sugere que para se obter um bom crescimento $\mathrm{e}$ desenvolvimento (altura da parte aérea e/ou diâmetro do coleto) de mudas de Anadenanthera peregrina, deve-se usar $100 \%$ de CAC na formulação do substrato, onde se alcançou valores satisfatórios do tratamento e T5=1,61 mm (100\% de CAC). Vallone et al. (2004) testando CAC em mudas de cafeeiro e Aguiar et al. (1992) trabalhando com mudas de Eucalyptus grandis observaram que o uso de 60 a $70 \%$ de CAC em substituição ao substrato comercial proporcionaram melhor desenvolvimento das mudas em viveiro. 


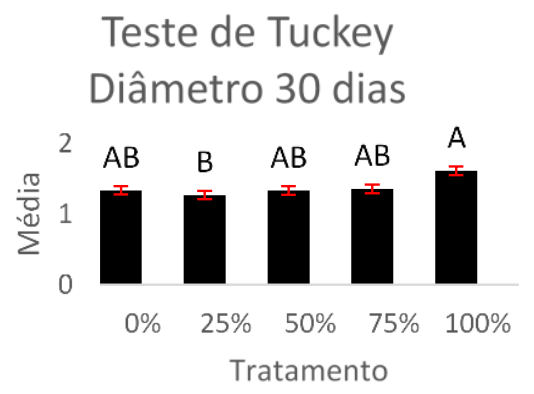

\section{Teste de Tuckey \\ Diamêtro 60 dias}

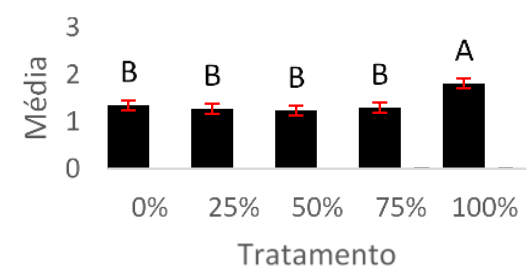

\section{Teste de Tuckey}

Diâmetro 90 dias

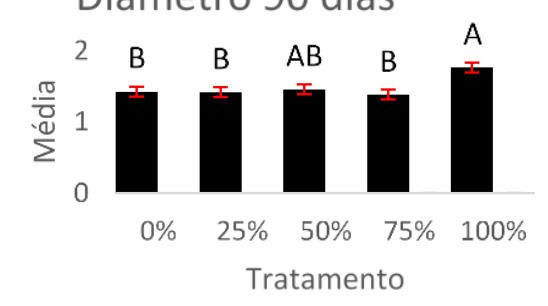

Figura 2. Teste de comparação de médias dos diâmetros aos 30, 60 e 90 DAE, médias seguidas pela mesma letra não diferem entre si, pelo teste de Tukey, a 5\%.

Quanto ao índice de qualidade de Dickson (IQD) obteve-se valores entre 0,035 a 0,139 onde o tratamento que apresentou os melhores resultados foi aquele no qual utilizou adição de $100 \%$ de CAC + 0\% de substrato comercial Bioflora ${ }^{\circledR}$ (Figura 3). Segundo Fonseca et al. (2002) e Delarmelina et al. (2015) o IQD é um bom indicador da qualidade das mudas, pois no seu cálculo são consideradas as robustezes e o equilíbrio da distribuição da biomassa na muda, ponderando os resultados de vários parâmetros importantes empregados para avaliação da qualidade. Entretanto quando confrontamos os dados do presente estudo com os valores propostos por Hunt (1990) que

Teste de Tuckey IQD 30 dias

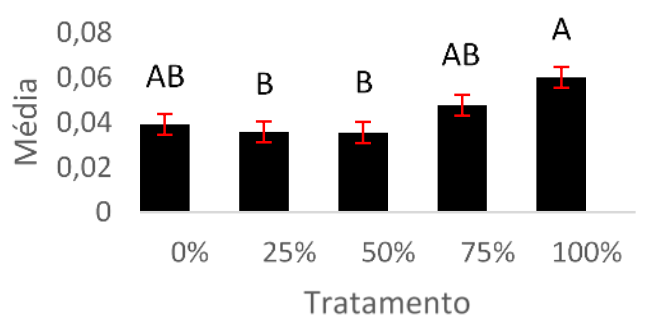

estabelece valor mínimo de 0,20 concluímos que todos os tratamentos testados não obtiveram valores satisfatórios (Figura 3).

De acordo com Clarkson (1985) e Faria et al. (2016) o IQD é comumente maior em ambiente de baixa fertilidade, podendo ser considerada uma estratégia da planta para retirar o máximo de nutrientes naquela condição. Dessa forma verificou-se que o IQD aumentava à medida que diminuía o suprimento de nutrientes, característica observada no tratamento T5 $(100 \%$ de CAC $+0 \%$ de substrato comercial Bioflora $^{\circledR}$ ).

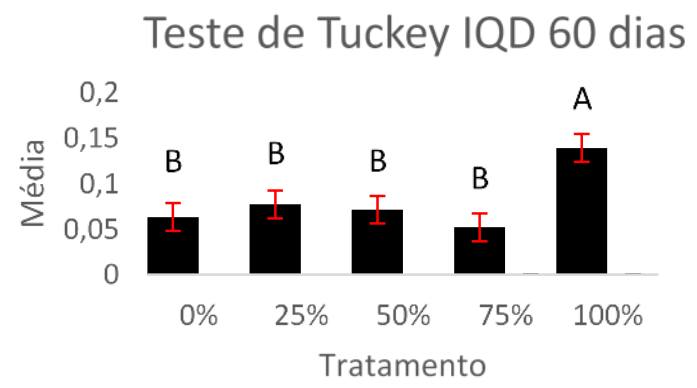

Figura 3. Teste de comparação de médias do IQD aos 30 e 60 DAE, médias seguidas pela mesma letra não diferem entre si, pelo teste de Tukey, a $5 \%$. 
Saidelles et al., (2009) observaram que a espécie de Enterolobium contortisiliquum utilizando os mesmos tratamentos do presente estudo, constataram que, o uso de $50 \%$ de CAC misturada ao solo foi o que apresentou melhores resultados, sendo assim recomendado para uso na produção de mudas de qualidade. Já o presente estudo observou-se que $100 \%$ de CAC sem mistura com solo ou substrato comercial foi o melhor tratamento testado, fato que pode ser explicado pelas características da espécie, ou seja, Anadenanthera peregrina ocorre em diferentes tipos de solos desde solos secos e úmidos, solos rasos, compactados e mal drenados, além de ser uma espécie de crescimento de rápido a moderado (Carvalho, 2003; Souza et al., 2012).

\section{CONCLUSÃO}

O substrato alternativo casca de arroz carbonizado, na porção de $100 \%$ proporcionou os melhores resultados nas variáveis e no índice de qualidade de Dickson.

Apesar de o tratamento T5 está proporcionando os melhores resultados, não é recomendado para a produção de mudas de Anadenanthera peregrina (L) Speg. pois as mesma não são classificas como mudas de boa qualidade, devido o índice de qualidade de Dickson ser inferior a 0,20 que é o mínimo recomendado pela literatura.

Todos os autores declararam não haver qualquer potencial conflito de interesses referente a este artigo.

\section{REFERÊNCIAS}

AGUIAR, I. B.; ISMAEL, J. J.; BANZATTO, D. A.; VALERI, S. V.; ALVARENGA, S. F.; CORRADINE, L. Efeitos da composição do substrato para tubetes no comportamento de Eucalyptus grandis shille x maiden no viveiro e no campo. Circular Técnica, Piracicaba: IPEF, n.180, p. 1-10, 1992.
ARAÚJO F. S.; MARTINS S.V.; MEIRA NETO J. A. A.; LANIS J. L. L.; PIRES I. E. Estrutura da vegetação arbustivo-arbórea colonizadora de uma área degradada por mineração de caulim, Brás Pires, MG. Revista Árvore, 2006; 30 (1): 107116.

BERLOFFA, J.M.; GRAICHEN, F. A. S.; FERNANDES, F. M.; DIAS, A. R. Qualidade fisiológica e sanitária de sementes de angico - vermelho sobre o crescimento inicial de plântulas. Revista de Ciências Agro-Ambientais, v. 13, p. 78-86, 2015.

BEZERRA, K. A.; SANCAO, M. G.; GONCALVES, D. S.; DE SOUZA, P. B. Casca de arroz carbonizada na composição de substratos para emergência e produção de mudas de Acacia mangium Willd.. DESAFIOS, v. 4, p. 25-30, 2017.

CARVALHO, P. E. R. Espécies arbóreas brasileiras. Colombo: Embrapa-CNPF; Brasilia, D.F.: EmbrapaSPI, 2003. 1039p.

CLARKSON, D. T. Adaptações morfológicas e fisiológicas das plantas a ambientes de baixa fertilidade. In: Simpósio sobre reciclagem de nutrientes e agricultura de baixos insumos nos trópicos, 1985, ilhéus. anais... ilhéus: ceplac/ sbsc, 1985. p. 45-75.

DANIEL, O.; VITORINO, A. C. T.; ALOISI, A. A.; MAZZOCHIN, L. TOKURA, A. M.; PINHEIRO, E. R.; SOUZA, E. F. Aplicação de fósforo em mudas de Acacia mangium. Revista Árvore, Viçosa, v. 21, n. 2, p. 163-168, 1997.

DELARMELINA, W. M.; CALDEIRA, M. V. W.; FARIA, J. C. T.; LACERDA, L. C. Uso de resíduo orgânico em substrato para produção de Chamaecrista desvauxii (Collad.) Killip var. latistipula (Benth.). CERNE, v. 21, p. 429-437, 2015.

DIAS, P. C.; XAVIER, A.; OLIVEIRA, L. S.; FÉLIX, G. A.; PIRES, I. E. Resgate vegetativo de árvores de Anadenanthera macrocarpa. CERNE (UFLA), v. 21, p. 83-89, 2015.

DICKSON, A.; LEAF, A. L.; HOSNER, J. F. Quality appraisal of while spruce and white pine seedling stock in nurseries. Forestry Chronicle, Canadá, v. 36, n. 1, p. 11-13, 1960.

DUARTE, M. L.; PAIVA, H. N.; ALVES, M. O.; FREITAS, A. F.; MAIA, F. F.; GOULART, L. M. L. Crescimento e qualidade de mudas de vinhático 
(Platymenia foliolosa Benth.) em resposta à adubação com potássio e enxofre. Ciência Florestal, Santa Maria, v. 25, n. 1, p. 221-229, 2015

FARIA, J. C. T.; CALDEIRA, M. V. W.; DELARMELINA, W. M.; ROCHA, R. L. F. Substratos alternativos na produção de mudas de Mimosa setosa Benth. Ciência Florestal, v. 26, p. 1075-1086, 2016.

FAVALESSA, M. Substratos renováveis e não renováveis na produção de mudas de Acácia mangium. 2011. 60f. (Engenharia Florestal) Universidade Federal do Espirito Santo, Jerônimo Monteiro, 2011.

FEITOSA, D. G.; MALTONI, K. L.; CASSIOLATO, A. M. R.; PAIANO, M. O. Crescimento de mudas de Gonçalo-Alves (Astronium fraxinifolium) sob diferentes fontes e doses de nitrogênio. Revista Árvore, Viçosa, v.35, n. 3, 2011.

FONSECA, É. P.; VALÉRI, S. V.; MIGLIORANZA, É.; FONSECA, N. A. N.; COUTO, L. Padrão de qualidade de mudas de Trema micrantha (L.) Blume, produzidas sob diferentes períodos de sombreamento. Revista Árvore, Viçosa, v. 26, n. 4, p. 515-523, 2002.

FREITAS, E. C. S.; PAIVA, H. N.; LEITE, H. G.; OLIVEIRA NETO, S. N. Crescimento e qualidade de mudas de Cassia grandis Linnaeus f. em resposta à adubação fosfatada e calagem. Ciência Florestal, v. 27, p. 509-519, 2017.

GOMES, J. M.; PAIVA, H. N. de. Viveiros Florestais: propagação sexuada. Viçosa: UFV, 2011. 116 p.

HUNT, G. A. Effect of styroblock design and Cooper treatment on morphology of conifer seedlings. In:

TARGET SEEDLING SYMPOSIUM, MEETING OF THE WESTERN FOREST NURSERY ASSOCIATIONS, GENERAL TECHNICAL REPORT RM-200, 1990, Roseburg. Proceedings... Fort Collins: United States Departament of Agriculture, Forest Service, 1990. p. 218-222.

KLEIN, V. A.; CAMARA, R. K.; SIMON, M. A.; DIAS, S. T. Casca de arroz carbonizada como condicionador de substrato. In: FURLANI, A. M. C. Caracterização, manejo e qualidade de substrato para produção de plantas. Campinas: Instituto Agronômico, 2002. 95p. (Documentos IAC, n. 70).

KLINK, C.A.; MACHADO, R.B. Conservation of the Brazilian Cerrado. Conservation Biology, v.19, n.3, p.707-713, 2005.
LORENZI, H. Árvores brasileiras: manual de identificação e cultivo de plantas arbóreas nativas do Brasil. 3. ed. Nova Odessa: Instituto Plantarum, 2009. v. 2384 p. il. color.

MELO, L. A.; PEREIRA, G. A.; MOREIRA, E. J. C.; DAVIDE, A. C.; SILVA, E. V.; TEIXEIRA, L. A. F. Crescimento de mudas de Eucalyptus grandis e Eremanthus erythropappus sob diferentes formulações de substrato. Floresta e Ambiente, v. 21, p. 234-242, 2014.

MINAMI, K. Produção de mudas de alta qualidade em horticultura. São Paulo: Fundação Salim Farah Maluf, 1995. 128p.

MINISTÉRIO DO MEIO AMBIENTE, O Bioma cerrado. Disponível em:<http://www.mma.gov.br/biomas/cerrado>.

Acesso em: 15 de março de 2016.

NAVROSKI, M.C.; NICOLETTI, M. F.; LOVATEL, Q. C.; PEREIRA, M. O.; TONETT, E. C; MAZZO, M. V.; MENEGUZZI, A.; FELIPPE, D. Efeito do volume do tubete e doses de fertilizantes no crescimento inicial de mudas de Eucalyptus dunnii Maiden. Agrarian, v. 9, p. 26-33, 2016.

PACHECO M.V.; MATOS V.P.; FERREIRA R.L.C.; FELICIANO A.L.C.; PINTO K.M.S. Efeito de temperaturas e substratos na germinação de sementes de Myracrodruon urundeuva Fr. All. (Anacardiaceae), Brasil. Revista Árvore, Viçosa, v. 30, n. 3, 2006.

RANIERI B.D.; LANA T.C.; NEGREIROS D; ARAÚJO L.M.; FERNANDES G.W. Germinação de sementes de Lavoisiera cordata Cogn. E Lavoisiera francavillana Cogn. (Melastomataceae), espécies simpátricas da Serra do Cipó, Brasil. Acta Botanica, Brasílica 2003; 17(4):523-530.

ROSA Jr., E.J.; DANIEL, O.; VITORINO, A.C.T.; SANTOS FILHO, V.C. Efeito de diferentes substratos sobre o desenvolvimento de mudas de Eucalyptus grandis Hill, em tubetes. Revista Ciência Agronômica, 1:18-22, 1998.

SAIDELLES, F.L.F.; CALDEIRA, M.V.W.; SCHIRMER, W.N.; SPERANDIO, H.V. Casca de arroz carbonizada como substrato para produção demudas de tamboril-da-mata e garapeira. Ciências Agrárias, Londrina, v. 30, suplemento 1, p. 11731186, 2009. 
SMIDERLE O.J; SALIBE A.B; HAYASHI A.H; MINAMI K. Produção de mudas de alface, pepino e pimentão em substratos combinando areia, solo e Plantmax ${ }^{\circledR}$. Horticultura Brasileira, 19: 253-257, 2001.

SOUZA, E.R.B.; NAVES, R.V.; CARNEIRO, I.F.; LEANDRO, W.M.; BORGES, J.D. Crescimento e sobrevivência de mudas de cagaiteira (Eugenia dysenterica DC) nas condições do cerrado. Revista Brasileira de Fruticultura, v. 24, n. 2, p.491-495, 2002.

SOUZA, P. B.; SOUZA, A. L.; COSTA, W. S; PELLOSO, R.; LANA, J. M. Florística e diversidade das espécies arbustivo-arbóreas regeneradas no subbosque de Anadenanthera peregrina (L.) Speg. CERNE, v. 18, p. 413-421, 2012.

TABAJARA, S.M.; COLÔNIA, E.J. Casca de arroz e meio ambiente. Revista Lavoura Arrozeira, Porto Alegre, v. 39, n. 369, p. 10-12, 1986.

VALLONE, H.S.; GUIMARÃES, R.J.; SOUZA, C.A.S.; CARVALHO, J.A.; FERREIRA, R. S.; OLIVEIRA, S. Substituição do substrato comercial por casca de arroz carbonizada para a produção de mudas de cafeeiro em tubetes na presença de polímero hidrorretentor. Ciência Agrotécnica, Lavras, v. 28, n. 3, p. 598-604, 2004.

VENDRUSCOLO, E. P.; MARTINS, A. P. B.; CAMPOS, L. F. C.; BRANDAO, D. C.; NASCIMENTO, L. M.; SELEGUINI, A. Produção de mudas de batata-doce de baixo custo em diferentes substratos e níveis de enfolhamento de estacas. REVISTA DE AGRICULTURA NEOTROPICAL, v. 4, p. 102-109, 2017. 